\title{
The Two Stage Crystal Collimator for RHIC *
}

\author{
R. P. Fliller III $\dagger$, A. Drees, D. Gassner, L. Hammons, G. McIntyre, D.Trbojevic, BNL, Upton, NY 11793 \\ V. Biryukov, Y. Chesnokov, V. Terekhov, IHEP, Protvino, Russia
}

Abstract

The use of a two stage crystal collimation system in the RHIC yellow ring is examined. The system includes a copper beam scraper and a bent silicon crystal. While scrapers were installed in both of the RHIC rings before the year 2000 run, the crystal is installed for the 2001 run in one ring only, forming a two stage collimation system there. We present simulations of the expected channeling through the bent silicon crystal for both protons and gold ions with various beam parameters. This gives a picture of the particle losses around the ring, and the expected channeling efficiency. These results are then used to optimize the beam parameters in the area of the crystal to obtain maximum channeling efficiency, minimize out-scattering in the secondary collimator, and reduce beam halo.

\section{INTRODUCTION}

In present day high energy colliders, the requirements of high luminosity and low backgrounds place strict requirements on the quality of the beams used. At facilities like RHIC, intra-beam scattering (IBS) [1] and other halo forming processes become a major concern [2]. Transverse beam growth not only leads to increased detector backgrounds, but also reduces dynamic aperture of the accelerator leading to particle losses at high beta locations. To minimize these effects, an efficient collimation system is needed.

The optics of two stage collimation systems have been reported numerous places [3]. The main disadvantage of the usual two stage system is that particles hitting the primary collimator with small impact parameters can scatter out of the material, causing a more diffuse halo. A secondary collimator is used to improve the performance, but the problem of small impact parameters can still exist. Using a bent crystal as the primary collimator in such a system, the channeled particles are placed into a well defined region of phase space. This allows the placement of a secondary collimator such that the impact parameters of the channeled particles are large enough to reduce the scattering probability, and most of the particles that hit the collimator are absorbed.

In this paper we present the layout of the crystal collimation system in RHIC. Following, we show results of simulations of the collimator and its effects in the RHIC yellow ring.

\footnotetext{
* Work performed under the auspices of the U.S. Department of Energy

† rfliller@bnl.gov
}

\section{COLLIMATION LAYOUT}

For the 2001 run, the yellow (counter-clockwise) ring has a two stage collimation system consisting of a $5 \mathrm{~mm}$ long crystal and a $450 \mathrm{~mm}$ long L-shaped copper scraper. Both are located in a warm section downstream of the IR triplet magnets in the 7 o'clock area. This location was chosen because it has a large beta function $\left(\beta_{x} \approx 370 \mathrm{~m}\right)$ and low dispersion function $(\mathrm{D} \approx 0.15 \mathrm{~m})$. This allows for excellent betatron collimation [4]. Figure 1 shows the lattice functions in the vicinity of the collimators.

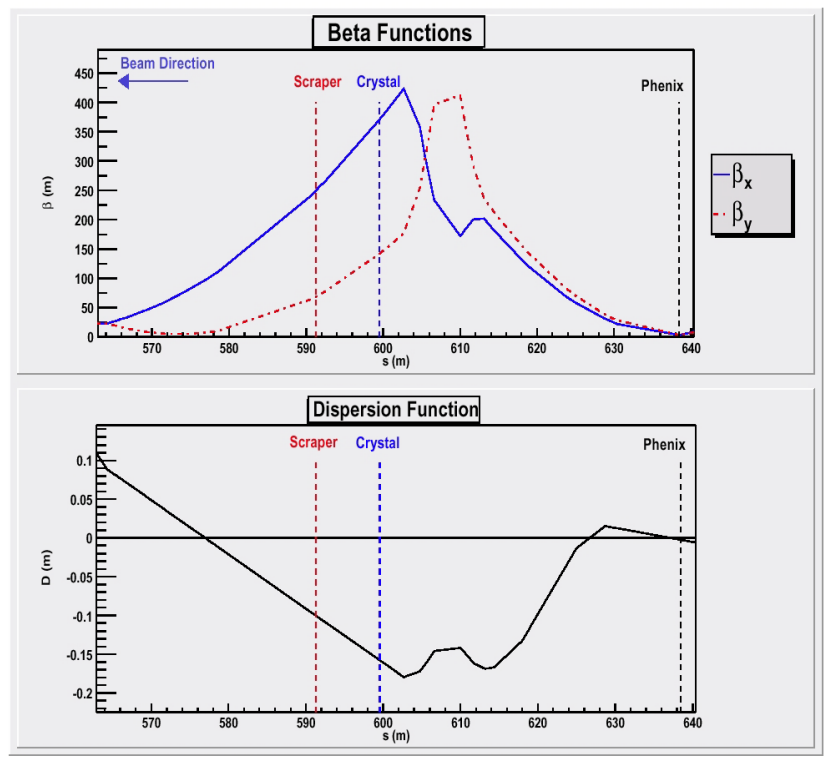

Figure 1: $\beta$ functions and dispersion function

The layout of the complete collimation system for RHIC is shown in figure 2. The crystal is an O-shaped silicon crystal with the (110) planes placed at an angle of $465 \mu \mathrm{rad}$ with respect to the normal of the input face, and a $0.44 \mathrm{mrad}$ bend. A schematic view is shown in figure 3. The choice of crystal dimensions is a result of previous simulations [5]. For channeling to occur in the crystal, the incoming angle of the particles must be less than the critical angle, $\theta_{c}$. The critical angle is the angle with respect to the crystal planes beyond which the particles cannot be channeled and is given by

$$
\theta_{c}=\sqrt{\frac{2 U\left(x_{c}\right)}{p v}}
$$

where $U\left(x_{c}\right)$ is the inter-planar potential at the location where the particle will enter the electron cloud of a lattice atom. For RHIC energies, $\theta_{c}=37 \mu \mathrm{rad}$ at injection and $11 \mu \mathrm{rad}$ at storage. 
Four PIN diodes are used to detect forward scattered particles from the scraper, and eight PIN diodes detect the back scattered particles as well as forward scattered particles from the crystal. A hodoscope consisting of two scintillators is aligned to detect scattered particles from the crystal. A drop in the count rate here is an indication of channeling. A phosphor screen is mounted on the scraper jaw to image the channeled particles.

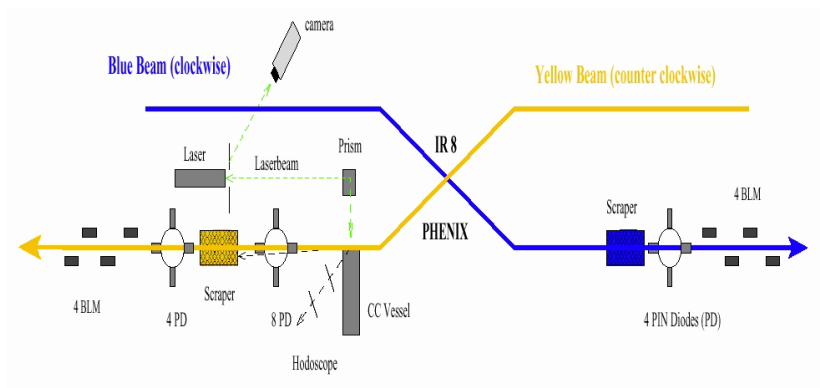

Figure 2: The RHIC collimation system

A stepper motor is used to control the motion of the crystal in the horizontal plane, with a maximum insertion of $1.27 \mathrm{~cm}$ from the beam pipe center. This corresponds to $5 \sigma$ and $3 \sigma$ of the beam size for gold and protons respectively. An inch-worm with a step resolution of $\approx 4 \mathrm{~nm}$ rotates the crystal with a step size of $\approx 0.013 \mu \mathrm{rad}$ over $15 \mathrm{mrad}$. A $0.5 \mathrm{~mW}$ Helium Neon (HeNe) laser placed $4.5 \mathrm{~m}$ from the crystal is reflected off the crystal side face to measure the crystal angle with respect to the beam pipe. Linear Voltage Differential Transformers (LVDTs) are used to monitor both the horizontal crystal position and the inch-worm motion. This, with the laser, gives a redundant measure of the angular position.

A more complete description of the collimation system is given in reference [6].

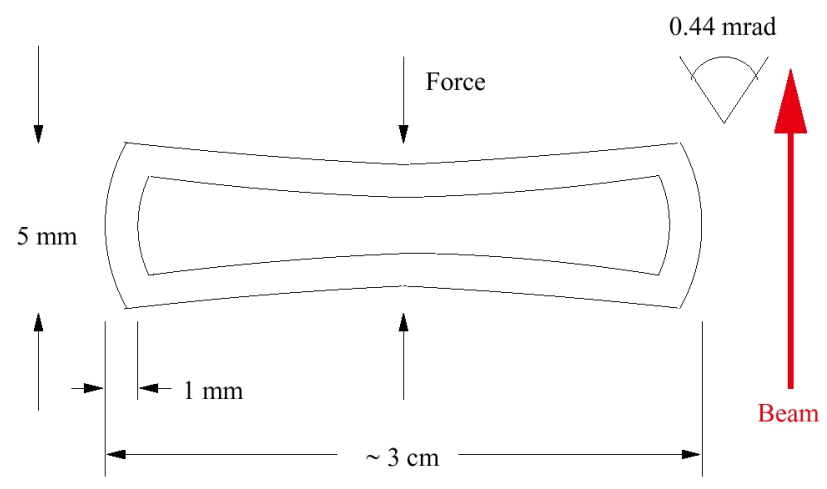

Figure 3: The RHIC Crystal

\section{SIMULATIONS}

The simulations of the collimation system included three major code components, UAL/TEAPOT for particle track- ing around the accelerator [7], $\mathrm{CATCH}$ [8] to simulate particle interactions in the crystal, and the K2 [9] code to implement the proton scattering in the copper jaw.

Gold ions and protons are tracked for 10 turns around the RHIC yellow ring, starting at the crystal. Particles that hit the crystal or the copper jaw are transfered to the proper program for simulation and then transfered back into TEAPOT to be tracked through the accelerator together with the noninteracting particles. In addition, the coordinates of each particle are saved at the entrance and exit of the crystal and scraper.

Ten thousand particles are tracked, uniformly filling a doughnut in $\mathrm{x}$ phase space so that particles would hit the crystal at all impact parameters. The y phase space is filled with an ellipse of area of $15 \pi \mathrm{mm}$ mrad. The maximum momentum deviation is 0.002 and no synchrotron motion is present. Typical initial transverse distributions are shown in figure 4. Coupling effects where not included to simplify the dynamics.
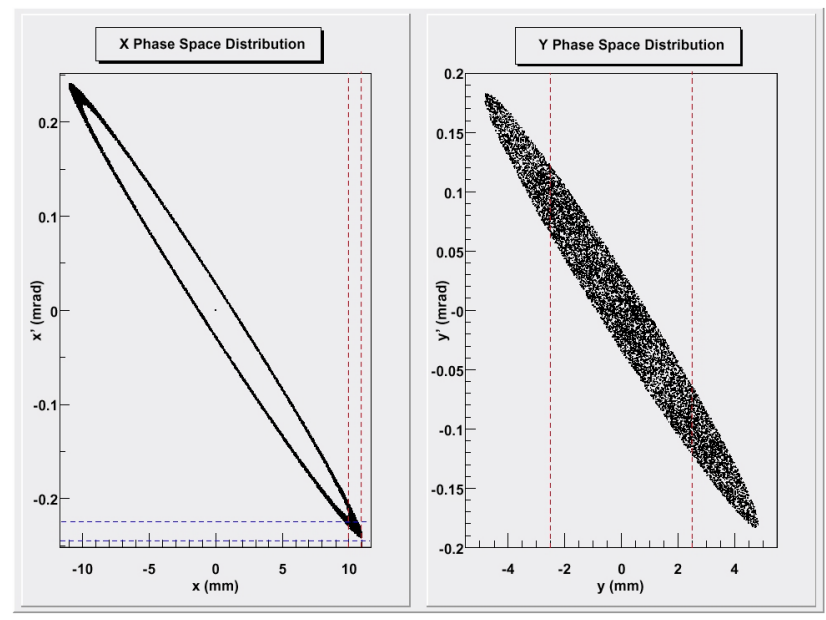

Figure 4: Transverse Phase Space Distributions. Vertical lines denote crystal position. Horizontal lines denote angular acceptance of crystal

In cases where the crystal is considered the only aperture limit and nonlinearities can be ignored, a transfer matrix is used instead of element-by-element tracking. The particle distribution is the same as above.

Figure 5 shows a typical phase space distribution for a perfectly aligned crystal. The left distribution is the crystal input distribution over 10 turns. The right distribution shows the output of the crystal. The dashed lines indicate the regions of crystal acceptance and emittance respectively.

Figure 6 shows the particle losses in the ring for a proton beam after 10 turns using only the crystal collimator. The crystal is at $5 \sigma$ and aligned with the beam envelope. 3254 protons entered the crystal, and $66 \%$ of the 1709 particles within the angular acceptance of the crystal where channeled. One can see that most of the particles hit the wall at Q5IT8 downstream of the crystal. The results for gold look similar. 

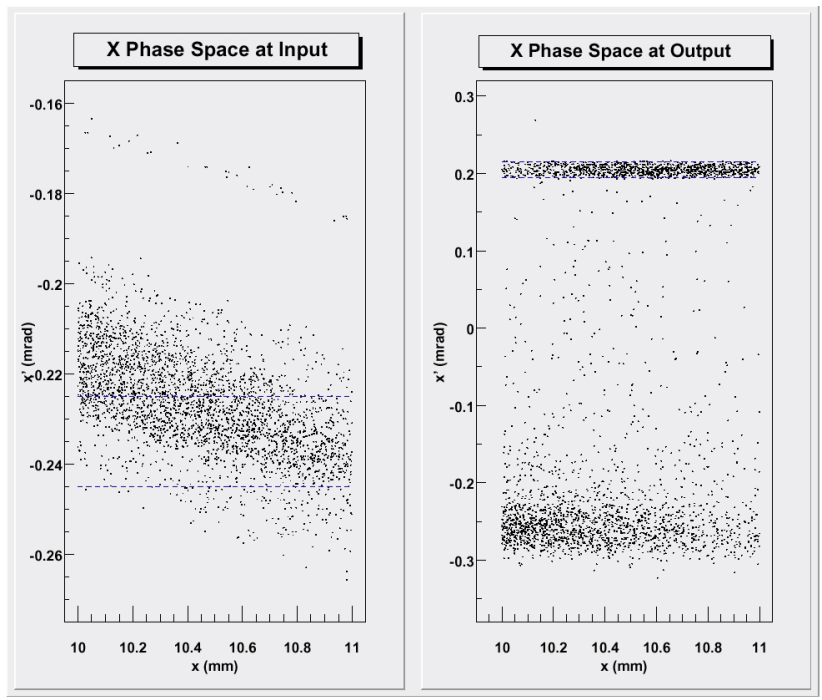

Figure 5: $\mathrm{X}$ phase space distribution at the crystal, overlaying 10 turns. The dashed lines denote the angular acceptance and emittance of the crystal respectively.

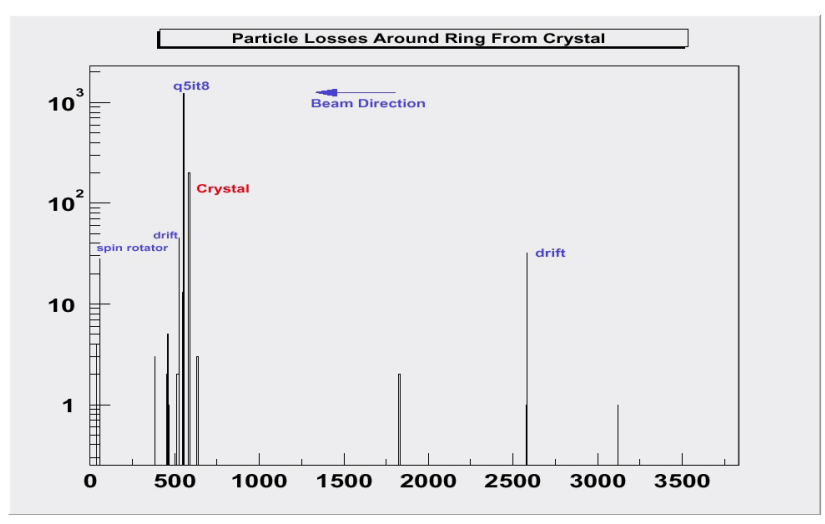

Figure 6: Particle Losses from Crystal

Figure 7 shows particle losses for the same alignment of the crystal and the $\mathrm{Cu}$ scraper inserted to $6 \sigma .3126$ protons hit the crystal, $63 \%$ of the 1836 protons within the angular acceptance of the crystal are channeled, 175 inelastically scatter and are lost. 2568 protons hit the scraper. All of the channeled protons hit the scraper the same turn. $95 \%$ of the protons that hit the scraper are inelastically scattered and lost. 165 of the protons that hit the crystal did not get lost. The overall efficiency of the system, defined as the ratio of protons that are lost in the collimators to the total number of protons lost, is $89 \%$. It is also obvious from figure 7 that the protons that escape the scraper are quickly lost because of their energy loss.

\section{FUTURE PLANS}

The commissioning of the crystal collimator will occur in the year 2001 run. Once the efficiency of the crystal collimator has been determined, a second apparatus will be built for the blue ring. That crystal has already been

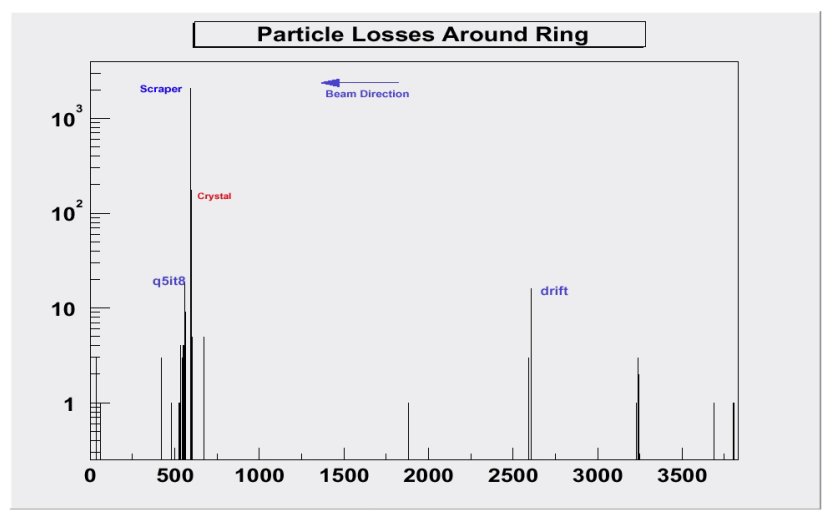

Figure 7: Particle Losses from Crystal and Cu Scraper.

manufactured. Once the collimator is commissioned it will be used in a variety of experiments to determine beam attributes such as size, angular profile, and diffusion rates.

In addition, there are plans to implement crystal extraction in the AGS for a neutrino mass experiment [10]. Experience gained at RHIC will be an important input for the development of that system.

We thank Nuria Catalán-Lasheras for her assistance with the $\mathrm{K} 2$ code.

\section{REFERENCES}

[1] J.Wei., "Intra Beam Scattering in RHIC", Report-no: RHIC/AP/63, July 1995

[2] N. Catalán Lasheras. "Transverse and Longitudinal Beam Collimation in a High-Energy Proton Collider (LHC)". Ph.D thesis, University of Zaragoza, 1998 (CERN-THESIS-2000019).

[3] T. Trenkler and J.B. Jeanneret., "The Principles of Two Stage Betatron and Momentum Collimation in Circular Accelerators" CERN Note: SL/95-03 (AP), LHC Note 312; M. Seidel. "The Proton Collimation System of HERA", Ph.D. thesis, Universität Hamburg, 1994 (DESY Report 94-103).

[4] D. Trbojevic, et. al. ,'A Study of Betatron and Momentum Collimators in RHIC" Report-No: RHIC/AP/142, November 1997

[5] V.M. Biryukov, et. al. "Optimization of the Parameters in the RHIC Single Crystal Heavy Ion Collimation”. PAC Proceedings (New York, 1999).

[6] The RHIC NIM paper here. Submitted to NIM.....

[7] N. Malitsky and R. Talman,"Unified Accelerator Libraries" CAP96; L. Schachinger and R. Talman, "A Thin Element Accelerator Program for Optics and Tracking", Particle Accelerators, 22, 1987.

[8] V. Biryukov, "Crystal Channeling Simulation - CATCH 1.4 User's Guide", SL/Note 93-74(AP),CERN 1993.

[9] T. Trenkler and J.B. Jeanneret. "K2. A software package for evaluating collimation systems in circular colliders." SL Note 94-105 (AP), December 1994.

[10] J.W. Glenn, K. Brown, and V. Biryukov "Continous Extracted Beam in the AGS Fast External Beam Line" these proceedings. 